\title{
Erratum
}

\section{Erratum to “A Primer on Magnetic Resonance-Guided La- ser Interstitial Thermal Therapy for Medically Refractory Epilepsy" by Lee EJ, et al. (J Korean Neurosurg Soc 62 : 353-360, 2019)}

https://doi.org/10.3340/jkns.2019.0105

In this article by Lee EJ et al., it was reported that the legend for Fig. 2 was incomplete. The complete figure legend is:

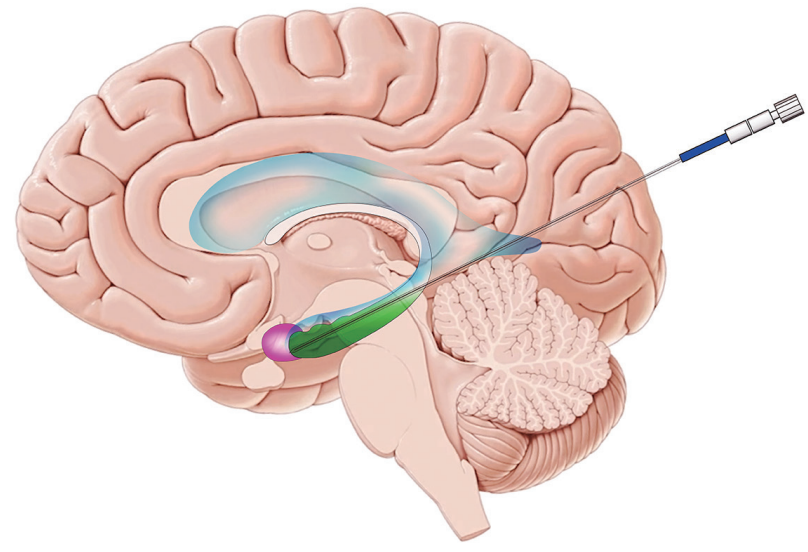

(A)
(B)

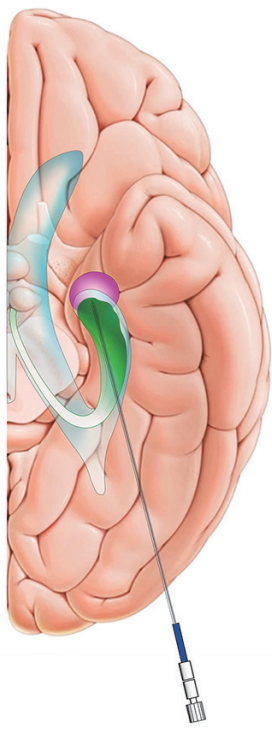

Fig. 2. Schematic depicting the target and the trajectory of laser probe for magnetic resonance-guided laser interstitial thermal therapy in patients with mesial temporal lobe epilepsy. Note the anatomical relationships between the ventricle (blue), hippocampus (green), and amygdala (pink). A : Sagittal view. B: Axial view. The reference of the background brain images is $\odot$ Kenhub (www.kenhub.com); Illustrator: Paul Kim. 